\title{
Integration of QMS and BSC based on Requirements
}

\author{
Gulmira Bazil, Nigina Toktassynova, Bakhyt Mukhanov, Kagazbek \\ Erenchinov, and Shynar Adilova
}

Engineering cybernetics, AUPET, Almaty, 050013, Kazakhstan

\author{
Keywords: Quality Management System (QMS), Balanced Scorecard (BSC), Integrated \\ Management System (IMS).
}

\begin{abstract}
Article is devoted to the integration of the requirements of quality management system and balanced scorecard for the implementation of integrated systems in the enterprise management. Effectiveness of the QMS is defined as the weighted partial criteria based on the prospects of BSC, which allows to measure the impact on the basis of numerical data
\end{abstract}

\section{Introduction}

Improving the efficiency of the enterprise depends on the quality of products and quality of control, so this issue is given great importance. The main problems associated with improving the quality management are included in the system of quality control mechanism for marketing activities; Orientation of quality management systems and all production activities to the consumer; strengthening mechanism of influence of quality management systems at all stages of the product life cycle. The methodological basis for the implementation of a quality management system is ISO 9001 defines the main directions of quality management, but in practice the results of implementation of the system are often unsatisfactory. Reasons for the low efficiency of most implemented and certified QMS enough, namely the unwillingness of top management to delve into the problem of quality management, incomplete implementation of the requirements of ISO 9001, lack of competence of the staff, the lack of quality objectives, and others. [1]. Besides, there is another reason for dissatisfaction leadership of quality management system, which is the difficulty in assessing the effectiveness of the QMS, i.e. shall be evaluated not only quantifiable indicators but also qualitative characteristics. Because of this is necessary to develop methods that would link all these aspects for certain specific outcome evaluation of QMS in the enterprise. In order to appropriately assess the performance of the QMS is proposed to supplement the financial indicators of non-financial [2], ie use a balanced scorecard (BSC) to assess the performance of the enterprise.

Based on the above, is currently the most effective way to improve the management of the enterprise is the implementation of Integrated Management Systems (IMS). Although BSC and quality management for a long time considered as a completely self-concept, the idea of integration itself is not new, similar proposals have repeatedly appeared on the pages of the magazine "Standards and Quality" because serious there is not contradictions between the ideology of the BSC and quality management. For example, in the BSC is managed by objectives. At the same time requirements for the determination of the quality objectives and aligning them with the overall objectives of the organization - one of the basic requirements of ISO 9001.

\section{System model of the requirements' complex}

Development of IMS in the enterprise is a long and difficult process that requires the involvement of all employees, but before it is necessary to begin to build a model of the system design and the steps to submit the final result. Therefore, the construction of IMS should begin immediately with the merger of the requirements of management systems and Balanced Scorecard (Figure 1). 


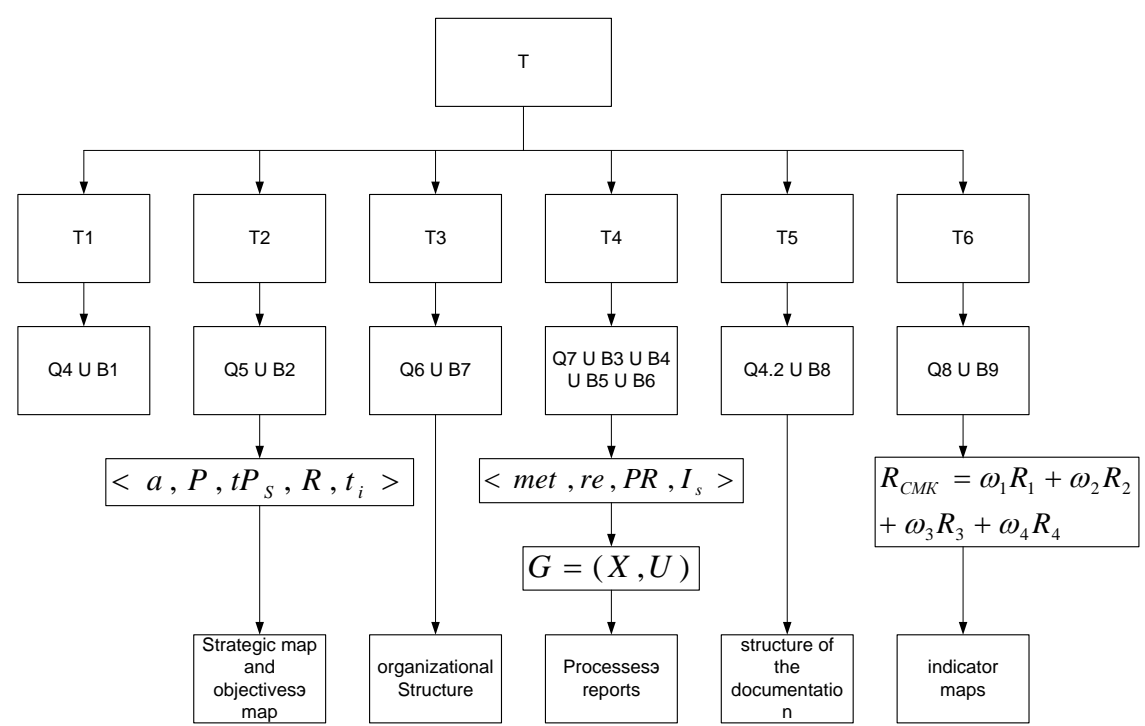

Fig. 1. System model of the requirements' complex of the QMS and BSC

System model of the requirements' complex of the integrated system obtained by the merger of the requirements for the BSC and QMS can be represented by the following equation:

$$
T=\cup T_{i} .
$$

$T_{i}$ - the set of all requirements for the integrated system; $U$ - a symbol of the union of sets of logic operation; $T_{1}$ - set of requirements for the integrated system as a whole; $T_{2}$ - set of requirements for building purposes; $T_{3}$ - set of requirements for the resource management; $T_{4}$ - set of requirements for the basic processes; $T_{5}$ - set of requirements for documentation; $T_{6}$ - set of requirements for the measurement, analysis and improvement.

Each of the sets $T_{i}$ requirements for the integrated system in Eq 1 is the union of the specific requirements of the relevant paragraphs of ISO 9001 and the requirements for the construction the BSC [3].

Integration set of requirements for the integrated system can be represented by:

$$
T_{1}=Q_{4} \cup B_{1} \text {. }
$$

$Q_{4}$ - Requirements paragraph 4 "Quality Management System" standard ST RK ISO 9001-2009; $B_{1}$ - developed requirements for BSC paragraph 1 "General Provisions" [3].

The set of requirements for construction purposes are defined as follows:

$$
T_{2}=Q_{5} \cup B_{2} \text {. }
$$

$Q_{5}$ - Requirements paragraph 5 "Management's Responsibility" standard ST RK ISO 9001-2009; $B_{2}$ - developed requirements for the BSC paragraph 2 "Goal Setting".

Integration requirements for resource management:

$$
T_{3}=Q_{6} \cup B_{7} \text {. }
$$

$Q_{6}$ - Requirements paragraph 6, "Resource management" standard ST RK ISO 9001-2009; $B_{7}-$ developed requirements for BSC paragraph 8, "The responsibility for achieving indicator values". Integration of requirements for the basic processes:

$$
T_{4}=Q_{7} \cup B_{3} \cup B_{4} \cup B_{5} \cup B_{6} \quad .
$$

$Q_{4}$ - Requirements paragraph 7 "Processes of product life cycle" standard ST RK ISO 9001-2009; $B_{3-7}-$ developed requirements for MSP paragraphs 3-6 "Prospects of development of the enterprise". Documentation requirements are determined by IMS through:

$$
T_{5}=Q_{4.2} \cup B_{8} .
$$

$Q_{4.2}$ - requirements paragraph 4.2 "Documentation Requirements" standard ST RK ISO 9001-2009; $B_{8}$ - developed requirements for BSC paragraph 9 "Map of scorecards". 
The set of requirements for measurement, analysis and improvement of IMS are defined through:

$$
T_{6}=Q_{8} \cup B_{9} .
$$

$Q_{8}$ - Requirements paragraph 8 "Measurement, analysis and improvement" standard ST RK ISO 9001-2009; $B_{9}$ - developed requirements for MSP paragraph 10 "Measurement, analysis, improvement."

\section{The implementation of the requirements for the purposes of ISM}

IMS development should begin immediately with the definition of a strategy or set long-term goals of the enterprise (the requirement $T_{2}$ ). When defining strategy should define the direction of the enterprise development, to create a processes according to the perspectives, create the necessary resources and determine the timing of its achievements. Therefore, goal-oriented system, which is IMS, may well be described by the following conceptual model:

$$
T_{2}=<a, P, t P_{S}, R, t_{i}>\text {. }
$$

$a$ - goal of system, $P$ - system development prospects, $t P_{S} \in T P$ - set processes involved in achieving, $R$ - resources to achieve the goals, $t_{i}$ - time to reach the goal. Implementation of the model (8) implementation requirements $T_{2}$ finally lead to the production of the main strategic objectives of the enterprisereflected in the strategic map of Norton and Kaplan [4]. Each goal should be to have his card, which will be reflected in the information of (8): name and value $a$, prospect of the goal $P$ (finance, customers, business processes, development and innovation), processes, which will lead to the implementation of the objectives $t P_{S}$, material and staff resources spent to achieve the goal $R$ and time $t_{i}$, for which this goal is to be achieved.

\section{Implementation of the process's requirements of the ISM}

None of goals can not be achieved without a clear vision of the business processes that are managed according to the ISO 9001 standard should be carried out using a process approach, which has a number of key advantages [5]. Process approach to management includes the development of many technological processes $t P_{S} \in T P$, involved in achieving the goals that are defined as [6 ]:

$$
t P_{S}=<m e t, r e, P R, I_{s}>
$$

$m e t$ - methods to achieve goals, re - means of achieving the goal, $P R$ - processing of information received in this class of systems, $I_{s}$ - processing of information received in the system. This method is used in the description of the construction of SADT-model, including elements of (9) and allowing to obtain high-quality reports, by the use of a graphical description of interrelated functional process models.

Using SADT-model in the construction process management allows to apply functional analysis of IMS (IMS method of representing functions of complex systems). Methodology of SADT-model is to construct models of two types: "as is" and "as it should be", that allows to realize a cycle of continuous improvement of the company. ISM process model can be represented by a directed graph:

$$
G=(X, U)
$$

$X$ - the set of vertices, reflects the process of product quality control, $U$ - set of arcs, reflecting connection between processes (documents, resources, information, products, effects), called data. Thus, the researcher has two graphs: $G 1=(X 1, U 1)$, displaying the process model "as is" and $G 2=(X 2, U 2)$ - "It should be". In the the compared sets, there are various elements that do not belong to both sets, as well as the same, belonging to both sets.

To determine the processes and data transferred from the model "as is" to the model "as it should be" necessary to find a subgraph $G 1_{A}=(A, U 3)$ of the graph $G 1=(X 1, U 1)$, which includes of the vertices (processes) of graph $G 1$, form a set $A$ with the arcs (data). 


$$
A=X 1 \cap X 2, \quad U 3=U 1 \cap U 2
$$

To get graph $G 2=(X 2, U 2)$ model "as it should be 'is necessary to define processes and data described by graph $G 4=(X 4, U 4)$, added to the subgraph $G 1_{A}=(A, U 3)$ to get the desired graph:

$$
X 2=A \cup X 4, \quad U 2=U 3 \cup U 4
$$

A list of newly MS quality processes and data is defined as the difference between the pairs of sets:

$$
X 4=X 2 \backslash A, \quad U 4=U 2 \bigcup U 3
$$

As a result of obtaining a graph $G 4=(X 4, U 4)$ the company defines steps to improve enterprise processes. The result of the implementation of the requirement $T_{4}$ is to obtain a clear vision of business processes, as a description of activities, using SADT allows to:

- set rules at all levels of management of enterprise;

- to solve the basic problem of regulation - lack of clarity and stiffness presentation process as a kind of sequence of procedures and actions to achieve this or other significant result, from the point of view of the enterprise, the result (s), thereby partially solved the problem with the documentation.

\section{Implementation of the documentation requirements of ISM}

Implementation of the documentation requirements $T_{5}$ necessary start by defining the structure of IMS (Figure 2), which combines document management system and balanced scorecard and identify lack or redundant information.

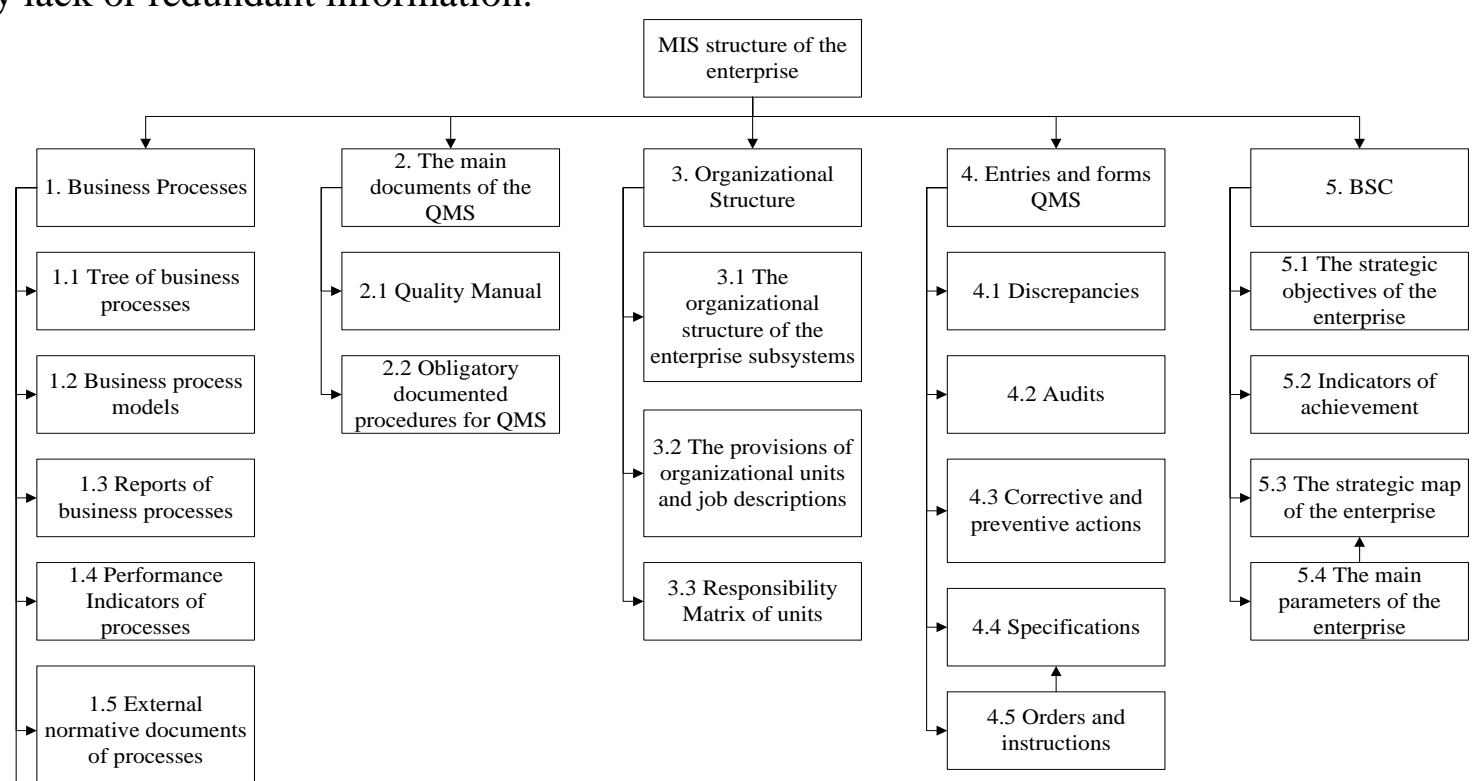

Fig. 2. Structure of the documentation IMS of Enterprise

\section{The implementation of the requirements for measurement, analysis and improvement of IMS}

Implementation requirements $T_{6}$ for measurement, analysis and improvement of includes:

- measurement strategy $B_{10}$;

- audits of processes functioning $Q_{8.22}$;

- $\quad$ analysis of the effectiveness of the leadership of the IMS $Q_{8.4}$ and ect.

The project of implementing IMS in scale of changes in many ways is similar to re-engineering of business: the similarity is that the first results of the project, as a rule, have no explicit financial and economic equivalent, ie, fall on non-financial area, for example, lead to a decrease in the number of errors and inconsistencies internal processes, improve staff competence and customer 
satisfaction, organizational culture change, etc. Therefore, an objective assessment of the effectiveness of the QMS conventional methods may be inappropriate [7].

For this days proposed many techniques based on different approaches to the performance assessment of individual elements of the system: processes [8], working with suppliers (logistics), evaluation of customer satisfaction and others. Unfortunately, a universal methodology for assessing the QMS does not exist, because of the system developed to whole company, so will assess the QMS through the achievement of objectives and indicators of processes. Determination of the effectiveness of the arithmetic mean of the weighted partial criteria:

$$
R_{Q M S}=\omega_{1} R_{1}+\omega_{2} R_{2}+\omega_{3} R_{3}+\omega_{4} R_{4}{ }^{1}
$$

$R_{1}$ - criterion that characterizes the financial component of the enterprise;

$R_{2}$ - criterion that characterizes customer satisfaction;

$R_{3}$ - criterion that characterizes the operation of business processes;

$R_{4}$ - criterion that characterizes the continuous development and employee engagement;

$\omega_{1} \ldots \omega_{4}$ - weights determined by the method of expert evaluations.

Each criterion can be calculated based on the strategic map: as within one perspective (criterion) there are independent development goals and objectives that depend on other purposes, so to calculate the criteria necessary to consider two cases:

- for independent goals $R_{\ell}=\frac{\sum_{i=1}^{n} G_{i}}{n}$

- for the goal depends on the prospects for other purposes within $R_{\ell}=\frac{\sum\left(\alpha_{j} \cdot G_{j i}\right)+G_{i}}{2}$,

$G-\%$ of achievement; $i$ - index dependent objective from $j ; \alpha$-impact objective weight $j$ to objective $i ; \ell=1 . .4$

Percent of achievement $G_{i}$ depends on the performance indicators:

$$
G_{i}=\sum \beta_{k}^{i} \cdot P_{k}^{i},
$$

$P_{k}^{i}-\%$ of achievement of the planned value of the k-th factors affecting to the goal $G_{i}$;

$\beta_{k}^{i}$ - weighting factor impact k-th indicator to achieve the objective $G_{i}$.

Achieving the planned value indicator $P_{k}^{i}$ calculated using the scorecard BSC (Table 1).

Table 1: Map of Scorecard

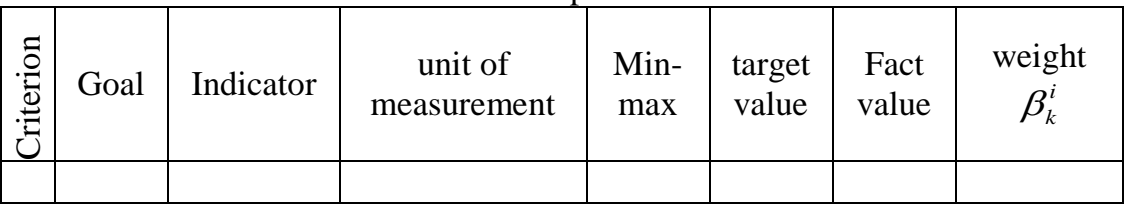

\section{Conclusions}

Thus considered the model of the complex system requirements for the integrated system possible to allocate following advantages of the introduction and development of IMS in the enterprise:

- Creation of IMS will provide greater coherence of the enterprise, thereby enhancing synergies;

- IMS minimizes the functional dissociation of the company arising in the development of autonomous control systems;

- combination of key procedures and documentation that allows you to organize the management process, reduce the number of documents and costs, in particular related to the elimination of the consequences of making a wrong decision;

- determine the effectiveness of the QMS using BSC, to avoid the use or implementation of other systems, and to make decisions to improve on the basis of numerical calculations.

${ }^{1}$ Note: The number of criteria may vary depending on the direction of enterprise development 


\section{Acknowledgements}

The research work was supported by Ministry of Education and Science under Grant No. 0044/GF.

\section{References}

[1] Egorov L. Reasons of inefficiency of quality management systems // Standards and Quality, № $12,2006$.

[2] Glitch A.V. Basics of quality control, 2nd ed. - M .: Standards and quality, 2001.

[3] Report on the research work: Research, development and implementation of scientific and methodological foundations of a BSC and software and hardware energy-efficient informationanalytical heat supply management system cities of Kazakhstan. Number 0044 / GF. Appendix G.2. Almaty, 2014.

[4] R.S.Kaplan, D.P. Norton. Balanced Scorecard from strategy to action. // - Moscow, p. 214, 2003.

[5] Report on the research work: Research, development and implementation of scientific and methodological foundations of a BSC and software and hardware energy-efficient informationanalytical heat supply management system cities of Kazakhstan. Number 0044 / GF. Appendix G. Almaty, 2014

[6] Khorosheva ER Theoretical bases of construction integrated systems management industry. // The thesis for the degree of Doctor of Technical Sciences. Vladimir, p. 328, 2007.

[7] Information on http://quality.eup.ru/MATERIALY14/ee-smk.htm

[8] Shkolina T.V. Scientific and methodological support of an integrated quality management system of the organization. // Thesis for the degree of candidate of technical sciences. Bryansk - p. 203, 2010. 